\title{
Metabolic abnormalities in adolescents with polycystic ovary syndrome in south china
}

\author{
Jia Huang ${ }^{1}$, Renmin $\mathrm{Ni}^{2}$, Xiaoli Chen ${ }^{1}$, Lili Huang ${ }^{1}$, Yaqin $\mathrm{Mo}^{1}$, Dongzi Yang ${ }^{1 *}$
}

\begin{abstract}
Background: Adults with polycystic ovary syndrome (PCOS) can have multiple metabolic abnormalities. However, studies in the adolescent population are still limited and these results seem to vary widely. This study was to investigate the metabolic abnormalities in adolescents with PCOS in South China and the potential risk factors contributed to these health risks.

Methods: Anthropometric measurements and biochemical parameters were evaluated in 128 adolescents with PCOS and their age- and BMI-matched controls.

Results: The prevalence of pre-diabetes, insulin resistance, hyperinsulinemia, dyslipidemia and metabolic syndrome in adolescents with PCOS was $11.7 \%, 46.9 \%, 29.7 \%, 22.7 \%$ and $4.7 \%$, respectively. $16.3 \%, 74.4 \%, 67.4 \%, 39.5 \%$ and $14 \%$ of the PCOS subjects with BMI > 85th had pre-diabetes, insulin resistance, hyperinsulinemia, dyslipidemia and metabolic syndrome, whereas 9.4\%, 32.9\%, 10.6\%, $14.1 \%$ and $0 \%$ of the PCOS subjects with BMI < 85th had such disturbances.

Conclusions: Adolescents with PCOS in South China had more metabolic abnormalities than their age- and BMImatched non-PCOS counterparts. Obesity could worsen insulin resistance, hyperinsulinemia and metabolic syndrome in PCOS adolescents.
\end{abstract}

\section{Background}

Polycystic ovary syndrome (PCOS) is a heterogeneous endocrine disorder that affects about 6\%-8\% women worldwide [1]. The consequences of the PCOS extend beyond the reproductive axis; women with the disorder are at substantial risk for developing metabolic abnormalities [2]. Perpetual sequence of hormonal and metabolic aberrations in PCOS patients may commence early, even in adolescent period, and extend throughout life.

Metabolic abnormalities, including pre-diabetes, dyslipidemia and metabolic syndrome (MS), have been widely studied in adult women with PCOS. Women with PCOS tend to have more than two to four times metabolic disturbances compared to those without PCOS [3-5]. However, there are few studies on the metabolic disturbances in adolescents with PCOS all over the world. Moreover, our previous studies also

\footnotetext{
* Correspondence: yangdz@mail.sysu.edu.cn

'Department of Obstetrics and Gynecology, Memorial Hospital of Sun Yat-

Sen University, Guangzhou, Guangdong, China

Full list of author information is available at the end of the article
}

found a lower prevalence of the abnormal glucose tolerance and MS in Chinese women [6,7]. Therefore, we sought to exhibit the metabolic abnormalities in adolescents with PCOS.

$40-60 \%$ of women with PCOS are overweight or obese. The role of obesity as a contributing factor in the development of PCOS has been widely accepted [8]. Body mass index (BMI) levels correlate with both body fat and concurrent health risks. Hence, we explored the relationship between BMI levels and metabolic abnormalities in Chinese adolescents with PCOS.

The aim of this study was to investigate the characteristics of metabolic abnormalities in adolescents with PCOS in South China, and to explore their potential risks, especially obesity. We sought to determine whether screening adolescents for metabolic abnormalities would benefit.

\section{Methods}

\section{Subjects and study design}

We performed a cross-sectional study of 128 adolescents with PCOS recruited from the gynecological outpatient

\section{C)


department of Memorial Hospital of Sun Yat-Sen University from January 2004 to December 2009. PCOS was defined by the Rotterdam 2003 criteria [2]. PCOS subjects should present at least two of the following criteria: oligo- and/or anovulation (i.e. $\leq 8$ menstrual periods in a year or menstrual cycles more than 35 days in length); clinical hyperandrogenism (i.e. acne or modified Ferriman-Gallwey scores $\geq 8$ ) and/or biochemical hyperandrogenism (i.e. serum total testosterone $(\mathrm{TT}) \geq 2.6$ $\mathrm{nmol} / \mathrm{l}$, free testosterone $(\mathrm{FT}) \geq 6.0 \mathrm{pg} / \mathrm{ml}$, TT and FT normal values were determined by the clinical laboratory of the gynecology department at the Memorial Hospital of Sun Yat-Sen University); and polycystic ovaries (i.e. the presence of $\geq 12$ follicles in each ovary measuring 2-9 $\mathrm{mm}$ in diameter) after exclusion of other etiologies (e.g. congenital adrenal hyperplasia, androgensecreting tumours and Cushing's syndrome). The Rotterdam criteria have expanded rather than replacing the $\mathrm{NIH}$ criteria, because all women diagnosable by the NIH 1990 criteria would also meet the Rotterdam definition. This study could analyze two subgroups of PCOS according to different diagnostic approach.

Age- and BMI-matched controls were recruited from a total of 654 adolescents visiting the health-care center for an annual routine check-up. They were asked to fill out a questionnaire on their menstrual cycle pattern during last year and received anthropometric measurements. In total, 593 (90.7\%) completed, among which 89.4\% (530) had normal menstruation (defined by average length of the menstrual cycle between 21-35 days, and menstrual period less than 7 days.), but only less than 100 adolescents participated in further testing. After exclusion of polycystic ovaries by transrectal ultrasound, 40 adolescents served as the age- and BMImatched controls.

This study was approved by the institutional review board of the Hospital. Informed consent was obtained either from a legal guardian of each subject less than 18 years old or from those subjects 18 years old or older. Any medications known to affect sex hormone, glucose or lipid metabolism were discontinued for at least a month, with the exception of oral contraceptives for 3 months before the study. All subjects did not use any hypolipidemic or anti-hypertensive drugs.

\section{Study protocols}

All subjects who were enrolled in the study had a medical history and underwent physical examination, including weight, height, waist and hip circumferences, blood pressure, modified Ferriman-Gallwey scores, and acne scores. All measurements were done by observers trained with the same standard protocols [9-11]: waist circumference was measured midway between the lowest rib and the iliac crest with the subject standing at the end of gentle expiration, and hip circumference at the greater trochanters; blood pressure was measured twice with mercury sphygmomanometer, with subjects seated quietly for at least 5 minutes, and the readings were averaged as the final value. Ferriman-Gallwey scores were assessed by at least two observers; Transrectal ultrasound examination was performed to evaluate the ovaries using a Toshiba Sonolayer SSA-220A (Toshiba, Tokyo, Japan) with a mechanical $6-\mathrm{MHz}$ probe. Adolescents with regularly menstruation were scanned in the early follicular phase (cycle days 3-5), while those with oligomenorrhea or amenorrhea were scanned either at random or between days 3 and 5 after a progestin-induced withdrawal bleeding.

After a 10-hour overnight fasting, blood samples were taken from adolescents between the first and fifth day of the menstrual period/withdrawal bleeding in order to measure PRL, LH, FSH, estradiol $\left(\mathrm{E}_{2}\right)$, total testosterone (TT), free testosterone (FT), sex hormone-binding globulin (SHBG), DHEAS, 17-OHP, TSH, and lipid profile. An oral glucose-tolerance test (OGTT) using $75 \mathrm{~g}$ of glucose was then performed, and blood samples were taken at 0,60 , and $120 \mathrm{~min}$ for glucose and insulin measurement. HOMA-IR was calculated as (fasting plasma glucose $(\mathrm{mmol} / \mathrm{l}) *$ insulin $(\mathrm{mU} / \mathrm{ml})) / 22.5$.

Impaired fasting glycaemia (IFG), impaired glucose tolerance (IGT) and diabetes mellitus (DM) were defined by the criteria proposed by the American Diabetes Association (ADA) [12]. Patients with IFG and/or IGT are now referred to as pre-diabetes [12]. Dyslipidemia was defined as having one or more of the following: $\mathrm{CHOL} \geq 6.0 \mathrm{mmol} / \mathrm{L}, \mathrm{TG} \geq 1.7 \mathrm{mmol} / \mathrm{L}, \mathrm{HDL}-\mathrm{C}<1.29$ $\mathrm{mmol} / \mathrm{L}$ and $\mathrm{LDL}-\mathrm{C} \geq 3.6 \mathrm{mmol} / \mathrm{L}$. Insulin resistance (IR) was defined as the HOMA-IR value $\geq 95^{\text {th }}$ percentile, and hyperinsulinemia (HIN) as the fasting insulin level $\geq 95^{\text {th }}$ percentile [13]. MS was defined by International Diabetes Federation (IDF) criteria for MS in children and adolescent $[14,15]$. Adult IDF criteria also apply to adolescents more than 16 years [15], if they had central obesity (waist circumference $\geq 80 \mathrm{~cm}$ ) plus two or more of the following four factors: 1) increased concentration of TG $\geq 1.7 \mathrm{mmol} / \mathrm{l} ; 2$ ) reduced concentration of HDL-C $<1.29 \mathrm{mmol} / \mathrm{l}$; 3) raised blood pressure: systolic pressure $\geq 130 \mathrm{mmHg}$ or diastolic pressure $\geq 85 \mathrm{mmHg}$ or treatment of previously diagnosed hypertension; and 4) increased fasting glucose level $\geq 5.6$ $\mathrm{mmol} / \mathrm{l}$ or previously diagnosed type 2 diabetes.

\section{Assays}

PRL, LH, FSH, $E_{2}$, and TT were measured by the chemiluminescence immunoassays Access 2 (Beckman, USA); FT, SHBG, DHEAS, and 17-OHP were measured by Access 2 ELISAs (Beckman, USA). TSH was measured using a chemiluminescence immunometric assay (Immulite 2000 
Analyzer; CPC, Los Angeles, CA, USA). Total cholesterol (CHOL), high-density lipoprotein cholesterol (HDL-C), low-density lipoprotein cholesterol (LDL-C) and triglyceride (TG) were measured using an enzymatic calorimetric method with the 7600 autoanalyzer (Hitachi 7600). Plasma glucose was measured using the glucose oxidase method (Hitachi 7600) and plasma insulin using a chemiluminescence immunometric assay and commercial kit (Immulite 2000 Analyzer; CPC).

\section{Statistical analysis}

All data were analyzed using SPSS version 13.0 (SPSS Inc., Chicago, IL, USA). We assessed the normality of the distribution of all continuous variables using the Kolmogorov-Smirnov test. Because the data were not normally distributed, continuous variables were presented as medians and the interquartile ranges. Medians between patients and controls were compared with the Mann-Whitney $U$ test. Pearson $\chi^{2}$ test was used to analyze the differences between the groups and obtain an odds ratio (OR). Statistical significance was set at $p<0.05$.

\section{Results}

\section{Demographics and anthropometrics}

In total, 128 adolescents were diagnosed as PCOS according to the Rotterdam criteria. Of these, $96.1 \%$ presented oligomenorrhea or amenorrhea, all persisting for 2 years post menarche; $83.6 \%$ presented ultrasonographic appearance of polycystic ovaries; $74.2 \%$ presented clinical and/or biochemical hyperandrogenism. In total, $70.3 \%$ (90/128) of patients had both androgen excess and ovulatory dysfunction and met the NIH 1990 criteria.

The comparison of demographic characteristics between adolescents with PCOS and controls was summarized (Table 1). No differences were found between the controls whether had given a blood sample or not, concerning age, BMI, duration of the period, and quantity of menstrual flow. There were no differences in age, menarche age, gynecological age, and BMI between the subjects with and without PCOS. Subjects with PCOS had increased waist circumference, higher FG scores and blood pressure. The subjects with PCOS defined by different criteria presented almost the same results when compared with controls, except for the waist/hip ratio.

Table 2 listed the hormonal and metabolic features of adolescents with or without PCOS. Subjects with PCOS displayed significantly higher LH, TT, FT, 2-h glucose, fasting insulin, 2-h insulin, and HOMA-IR, but there were no significant differences in $\mathrm{FSH}$, fasting glucose, TG, CHOL, LDL and HDL between the PCOS and the control groups.
Table 1 Demographic characteristics of adolescents with and without PCOS

\begin{tabular}{cccc}
\hline & & \multicolumn{2}{c}{ PCOS } \\
& Control & Rotterdam criteria & NIH criteria \\
\hline $\begin{array}{c}\text { Number of } \\
\text { subjects }\end{array}$ & 40 & 128 & 90 \\
Age(yr) & $19.0(17.0-19.0)$ & $18.0(17.0-19.0)$ & $18.0(17.0-19.0)$ \\
Menarche(year) & $12.5(12.0-13.0)$ & $12.0(12.0-13.0)$ & $12.0(12.0-13.0)$ \\
Gynecological & $6.0(4.0-7.0)$ & $6.0(5.0-7.0)$ & $6.0(5.0-7.0)$ \\
age(year) & & & \\
BMI(kg/m2) & $19.6(18.4-21.0)$ & $20.0(18.5-25.1)$ & $20.2(18.4-25.2)$ \\
Waist(cm) & $67.3(63.0-72.8)$ & $72.0(67.5-80.0) * *$ & $72.0(68.0-80.4) * *$ \\
WHR & $0.78(0.75-0.81)$ & $0.80(0.76-0.84)$ & $0.80(0.76-0.85) *$ \\
SBP(mmHg) & $100.0(92-105)$ & $110.0(100-120) * *$ & $110.0(98-120){ }^{* *}$ \\
DBP(mmHg) & $60.0(60-66)$ & $71.0(65-78) * *$ & $71.0(65-78) * *$ \\
FG scores & $0(0-5.5)$ & $9(6-13) * *$ & $10(7-13) * *$ \\
\hline
\end{tabular}

Variables were expressed as median with (interquartile range).

Gynecological age = calendar age - menarche age; BMI: body mass index; WHR: waist to hip ratio; SBP: systolic pressure; DBP: diastolic pressure; FG scores: Ferriman-Gallwey scores.

${ }^{*} \mathrm{P}<0.05,{ }^{* *} \mathrm{P}<0.01$ vs. control.

Prevalence of metabolic abnormalities in adolescents with and without PCOS

The prevalence of various metabolic abnormalities in adolescents with and without PCOS was listed in Table 3. No DM was detected either in the PCOS group or in the control group. Pre-diabetes (IFG and/or IGT) was only found in the PCOS group, and the prevalence of

Table 2 Hormonal and metabolic features of adolescents with and without PCOS

\begin{tabular}{|c|c|c|c|}
\hline & \multirow[b]{2}{*}{ Control } & \multicolumn{2}{|c|}{ PCOS } \\
\hline & & $\begin{array}{l}\text { Rotterdam } \\
\text { criteria }\end{array}$ & NIH criteria \\
\hline FSH (IU/L) & $5.3(4.4-6.6)$ & $5.6(4.5-6.9)$ & $5.6(4.5-6.9)$ \\
\hline LH (IU/L) & $5.1(3.6-7.5)$ & $11.0(5.9-16.1) * *$ & $10.3(6.3-16.2)$ ** \\
\hline Tा (nmol/L) & $1.7(1.5-2.4)$ & $2.5(2.0-3.2) * *$ & $2.8(2.3-3.3) * *$ \\
\hline $\mathrm{FT}(\mathrm{pg} / \mathrm{ml})$ & $2.9(2.1-3.5)$ & $4.6(2.6-7.3) * *$ & $5.1(2.9-8.1) * *$ \\
\hline $\begin{array}{l}\text { Fasting glucose } \\
\qquad(\mathrm{mmol} / \mathrm{L})\end{array}$ & $4.7(4.5-4.8)$ & $4.8(4.5-5.0)$ & $4.8(4.5-5.1)$ \\
\hline $\begin{array}{l}\text { 2-h glucose } \\
(\mathrm{mmol} / \mathrm{L})\end{array}$ & $5.6(5.0-6.3)$ & $6.3(5.1-7.1) *$ & $6.3(5.3-7.1) * *$ \\
\hline $\begin{array}{l}\text { Fasting insulin } \\
\qquad(\mu \mathrm{U} / \mathrm{ml})\end{array}$ & $5.7(4.4-8.3)$ & $8.8(5.7-13.2) * *$ & $9.0(5.9-13.2) * *$ \\
\hline $\begin{array}{l}\text { 2-h insulin } \\
(\mu \mathrm{U} / \mathrm{ml})\end{array}$ & $48.1(35.5-82.4)$ & $72.7(46.6-131.9)^{*}$ & $76.7(51.0-139 .)^{* *}$ \\
\hline HOMA-IR & $1.2(0.9-1.8)$ & $1.9(1.2-2.9) * *$ & $1.9(1.4-2.9) * *$ \\
\hline $\mathrm{CHOL}(\mathrm{mmol} / \mathrm{L})$ & $4.2(3.7-4.7)$ & $4.5(4.1-5.1)$ & $4.5(4.1-5.1)$ \\
\hline TG (mmol/L) & $0.9(0.7-1.2)$ & $1.0(0.8-1.3)$ & $1.0(0.7-1.3)$ \\
\hline HDL (mmol/L) & $1.6(1.4-1.7)$ & $1.6(1.4-1.9)$ & $1.5(1.3-1.9)$ \\
\hline LDL (mmol/L) & $2.6(2.1-2.9)$ & $2.5(2.1-3.1)$ & $2.5(2.1-3.2)$ \\
\hline
\end{tabular}

Variables were expressed as median with (interquartile range).

FSH: follicle stimulating hormone; LH: luteinizing hormone; $\Pi$ : total

testosterone; FT: free testosterone; HOMA-IR: Homeostasis Model Assessment of Insulin Resistance; CHOL: total cholesterol; TG: triglyceride; HDL: highdensity lipoprotein cholesterol; LDL: low-density lipoprotein cholesterol. ${ }^{*} \mathrm{P}<0.05,{ }^{* * P}<0.01$ vs. control. 
Table 3 Prevalence of various metabolic abnormalities in adolescents with and without PCOS

\begin{tabular}{cccc}
\hline & \multicolumn{3}{c}{ PCOS (\%) } \\
& Control (\%) & Rotterdam criteria & NIH criteria \\
\hline IFG & 0 & 1.6 & 2.2 \\
IGT & 0 & $10.2^{*}$ & $12.2^{*}$ \\
Pre-diabetes & 0 & $11.7^{*}$ & $14.4^{* *}$ \\
HIN & 7.5 & $29.7^{* *}$ & $33.3^{* *}$ \\
IR & 17.5 & $46.9^{* *}$ & $44.4^{* *}$ \\
Hypercholesterolemia & 0 & 4.7 & 5.6 \\
Elevated LDL & 0 & 4.7 & 5.6 \\
Low HDL & 15.0 & 14.1 & 16.7 \\
Hypertriglyceridemia & 7.5 & 10.2 & 12.2 \\
Dyslipidemia & 17.5 & 22.7 & 26.7 \\
Central obese & 5.0 & $20.3^{*}$ & $24.4^{* *}$ \\
Raised BP & 2.5 & 8.0 & 8.2 \\
MS & 2.5 & 4.7 & 6.7 \\
\hline
\end{tabular}

IFG: impaired fasting glycaemia; IGT: impaired glucose tolerance; pre-diabetes: IFG and/or IGT; HIN: hyperinsulinemia; IR: insulin resistance; MS: metabolic syndrome.

${ }^{*} \mathrm{P}<0.05,{ }^{* * P}<0.01$ vs. control.

IFG, IGT and pre-diabetes was $1.6 \%, 10.2 \%$ and $11.7 \%$, respectively. The prevalence of hyperinsulinemia and insulin resistance raised more than 2.5 folds in PCOS group (29.7\% vs. $7.5 \% ; 46.9 \%$ vs.17.5\%). PCOS group also presented higher prevalence of central obese $(20.3 \%$ vs. $5 \%$ ). However, there were no significant differences in lipid disturbances and other components of MS between groups. The characteristics were consistent, no matter which diagnostic criteria were used to define PCOS.

Prevalence of metabolism abnormalities in different BMI subgroups of adolescents with PCOS

Of the sample of 530 otherwise healthy adolescents, the $85^{\text {th }}$ and $95^{\text {th }}$ percentile of BMI was $21.4 \mathrm{~kg} / \mathrm{m}^{2}$ and $23.8 \mathrm{~kg} / \mathrm{m}^{2}$, respectively. $33.6 \%(43 / 128)$ subjects with PCOS by Rotterdam criteria had a higher BMI above the $85^{\text {th }}$ percentile, and $36.7 \%$ (33/90) subjects by NIH criteria.

To assess the effect of BMI on the prevalence of prediabetes, dyslipidemia and metabolic syndrome, the subjects with PCOS were then divided into two groups, group $\mathrm{BMI} \geq 85^{\text {th }}$ and group $\mathrm{BMI}<85^{\text {th }}$. Although there was no statistical significance in pre-diabetes and hypercholesterolemia between the two groups (Table 4), group $\mathrm{BMI} \geq 85^{\text {th }}$ had higher risk of hyperinsulinemia (67.4\% vs. 10.6\%; OR, 17.5; 95\%CI, 6.8-44.8), insulin resistance (74.4\% vs. $32.9 \%$; OR, 5.9; 95\% CI, 2.6-13.5), and dyslipidemia (39.5\% vs. $14.1 \%$; OR, 4.0 ; $95 \% \mathrm{CI}, 1.7-$ 9.4) than those of group BMI $<85^{\text {th }}$ (Figure 1).
Table 4 Prevalence of metabolic abnormalities in adolescents with PCOS categorized by different BMI

\begin{tabular}{ccccc}
\hline & \multicolumn{2}{c}{ Rotterdam criteria } & \multicolumn{2}{c}{ NIH criteria } \\
BMI & $<$ 85th & $\mathbf{2}$ 85th & $<$ 85th & $\geq$ 85th \\
\hline Number of subjects & 85 & 43 & 57 & 33 \\
IFG & 2.4 & 0 & 3.5 & 0 \\
IGT & 7.1 & 16.3 & 10.5 & 15.2 \\
pre-diabetes & 9.4 & 16.3 & 14.0 & 15.2 \\
HIN & 10.6 & $67.4^{* * *}$ & 12.3 & $69.7^{* * *}$ \\
IR & 32.9 & $74.4^{* * *}$ & 28.1 & $72.7^{* * *}$ \\
Hypercholesterolemia & 2.4 & 9.3 & 3.5 & 9.1 \\
Elevated LDL & 1.2 & $11.6^{*}$ & 1.8 & 12.1 \\
Low HDL & 8.2 & $25.6^{* *}$ & 10.5 & $27.3^{*}$ \\
Hypertriglyceridemia & 3.5 & $23.3^{* *}$ & 3.5 & $27.3^{* *}$ \\
Dyslipidemia & 14.1 & $39.5^{* *}$ & 17.5 & $42.4^{*}$ \\
Central obese & 0 & $60.5^{* * *}$ & 0 & $66.7^{* * *}$ \\
Raised BP & 0 & $19.4^{* *}$ & 0 & $18.5^{*}$ \\
MS & 0 & $14.0^{* *}$ & 0 & $18.2^{* *}$ \\
\hline
\end{tabular}

Abbreviations as in Table 3.

${ }^{*} \mathrm{P}<0.05,{ }^{* *} \mathrm{P}<0.01,{ }^{* * * P}<0.001$ vs. the "BMI $<85$ th" value.

Moreover, metabolic syndrome was only detected in group BMI $\geq 85^{\text {th }}$.

\section{Discussion}

PCOS can have multiple metabolic disturbances, such as insulin resistance and compensatory hyperinsulinemia, impaired glucose tolerance, dyslipidemia and metabolic syndrome [16]. Concerning the long-term health risk, it is necessary to identify such metabolic disorders in those adolescents with PCOS as early as possible. However, data on metabolic disturbances in adolescents with PCOS are limited, and the results seemed to vary in studies of different populations. In this study, we first

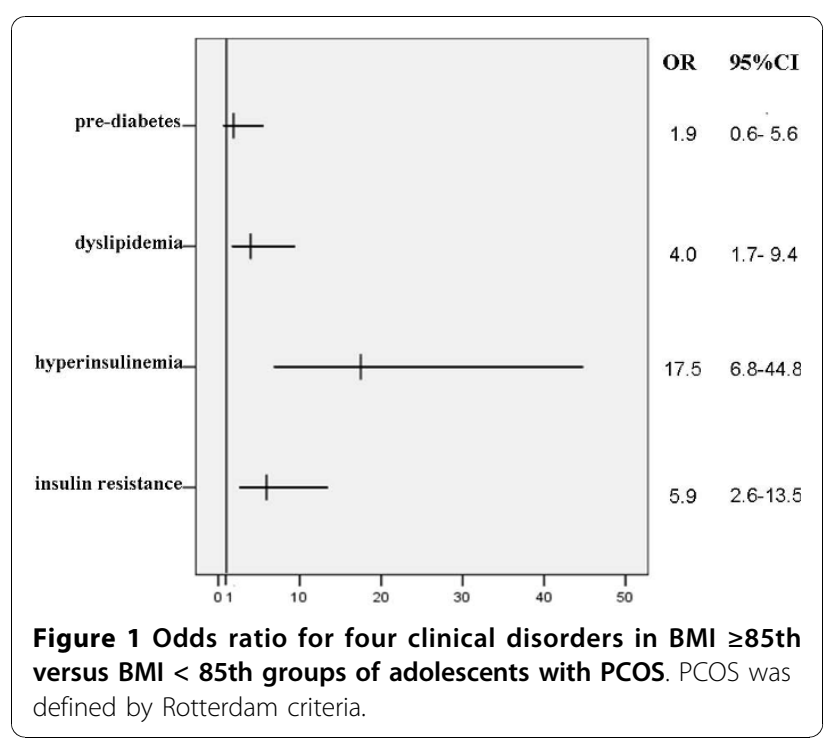


outlined the metabolic disturbances in Chinese adolescents with PCOS, and then we also studied the potential risk factors in such population.

Our data showed that the prevalence of IFG and IGT was $1.6 \%$ and $10.2 \%$, respectively. No T2DM was detected. A Canadian study of 22 obese adolescents with PCOS showed only one patient (4.5\%) suffered from IGT [17]. In contrast, two American studies from a similar geographic background population both indicated a higher prevalence of IGT in obese adolescents with PCOS (29.6\%, 8 in 27 [18]; 27.3\%, 3 in 11 [19], respectively). Considering the confounding effects of ethnicity and geographical background on the prevalence of metabolic disturbances, we also searched literatures published by Asian researchers, and only found one Korean study in which 1 in 16 (6.3\%) adolescents with PCOS had IGT [20]. In fact, the variations among these studies may due to the small sample size of subjects. However, a study report of 101 overweight Hispanic adolescents revealed that the prevalence of IGT and DM was $6.6 \%$ and $2 \%$, respectively [21]. In our study, the prevalence of IGT among overweight (BMI $\geq 85^{\text {th }}$ according to pediatric criteria [22]) subjects with PCOS was $16.3 \%$. Considering more detail of the different study population, the variation may due to the elder age in our study population.

Data from Nur et al [21] and our study indicated adolescents with PCOS had a lower prevalence of either pre-diabetes or T2DM than their same ethnicity and geographical background adults. The prevalence of IGT was $31-35 \%$ in adults, while DM was $7.5-10 \%[23,24]$. Although in our previous study the prevalence in Chinese adults with PCOS was lower (IGT 20.5\%, T2DM1.9\%) than that of American PCOS [6], the prevalence was still higher than that of Chinese adolescents. Given that the conversion from IGT to T2DM is substantially enhanced in women with PCOS $[25,26]$, the early identification of affected patients and institution of lifestyle changes or drug treatments may benefit. Like other studies, we noted that the fasting glucose failed to predict the majority of the subjects with IGT, confirming the recommendation that the most reliable screening test for IGT in PCOS adolescents is the 2-h OGTT after a 75 g glucose load [3].

The prevalence of MS, diagnosed by IDF criteria, was 4.7\% in the studied Chinese adolescents with PCOS. Metabolic disorders were common in these adolescents as more than one third of them exhibited at least one component of MS. Central obesity and dyslipidemia were the two most common metabolic features, which was also the case in adult PCOS patients according to our previous study [7]. A study of 49 white of nonHispanic adolescents with PCOS revealed the prevalence of MS was $37 \%$; the mean BMI of such population was
$32 \mathrm{~kg} / \mathrm{m}^{2}$ [27]. In India, 17 in 39 (43.6\%) subjects with mean BMI $29.3 \mathrm{~kg} / \mathrm{m}^{2}$ suffered from MS [28]. While the subjects with BMI $25 \mathrm{~kg} / \mathrm{m}^{2}$, the prevalence reported in Italy was $9.4 \%$ (5 in 53) [29]. Taken together all these results evidence that the MS can be found in PCOS women as early as in adolescence, however, the exact prevalence is not sure. The difference may due to several factors. First, there are no consistent criteria for the diagnosis of MS in adolescents. Important demographic and clinical differences exist in the typology of MS, depending on the definition [30-32]. Second, the ranges of age in these studies are quite different. Age is regarded as an important risk factor for MS in both the general population and PCOS patients. Data of our previous study indicated the increasing trend in the prevalence of MS [7]. Moreover, age could affect the diagnosis of PCOS, because of the physiologic irregular ovulation, insulin resistance, and multi-follicular ovarian morphology characteristic of normal adolescents. Thus, the diagnosis of PCOS in young adolescents should be prudent $[33,34]$. Third, obesity is an early step in the etiological cascade leading to full metabolic syndrome. Increased body weight in female significantly increases the risk of having MS [35]. Difference BMI levels could account for variations in the prevalence of MS. Other factors, such as sample size, genetic factors, dietary, exercise and geographic location may also have some effects.

In this study, the characteristics of metabolic disturbances also exhibited according to different diagnosis criteria for PCOS. Specialized criteria for the diagnosis of PCOS in adolescents have not been established yet, and instead, the diagnostic criteria for adults are used in clinical practice. At present, the 2003 Rotterdam criteria are more widely accepted than the NIH criteria, but the former tends to expand rather than replace the latter because all women diagnosed by the NIH 1990 criteria would also meet the Rotterdam criteria. The risk of metabolic disturbances may vary among different phenotypes of PCOS based on the Rotterdam criteria. Adults PCOS defined by NIH criteria had more severe metabolic abnormalities than those defined by Rotterdam criteria. The difference of prevalence of obesity and androgen excess may attribute to the characteristic [36-38]. In an attempt to avoid erroneous diagnoses, we decided to enroll female adolescents with more than two gynecologic years because a normalization of menstrual cycles mainly occurs within the first 2 years after menarche $[39,40]$. In our group, the median gynecologic age was 6 years. There were no significant differences in metabolic abnormities between the two groups categorized by two different criteria. In total, $70.3 \%$ (90 in 128) subjects met both the criteria. The number of non-NIH subjects group was small, which may weaken the power to detect the differences of metabolic features. 
Moreover, there were similar levels of BMI, FG score, TT and FT between the Rotterdam group and NIH group. Considering the effects of BMI and androgen on metabolic abnormities, it was not surprising to fail to detected the differences in the two groups PCOS.

Nearly $50 \%$ of PCOS patients suffer from obesity, although it is not included in the diagnostic criteria. Obesity contributes to the manifestations of PCOS by increasing the magnitude of hyperandrogenism and the rates of anovulatory cycles and infertility. The pathophysiologic mechanism is related to hyperinsulinemia which, in turn, is induced by insulin resistance [41]. Although many patients with PCOS had insulin resistance independent of obesity $[42,43]$, the obesity indeed worsen underlying insulin resistance and insulin resistance-associated reproductive and metabolic features. Our study suggested that, when matched for obesity, PCOS was associated with increased risks of insulin resistance, hyperinsulinemia and pre-diabetes, but was not associated with an increased risk of MS. Further analysis of PCOS subjects revealed that obesity was associated with increased risks of insulin resistance, hyperinsulinemia and MS, but was not associated with pre-diabetes. The PCOS status and obesity are both associated with the higher prevalence of insulin resistance and hyperinsulinemia. As a central pathogenetic feature of PCOS, insulin resistance could be worsened by obesity. And the impact of obesity is a more significant contributor to the prevalence of MS than PCOS status. In a word, our study emphasized the importance of PCOS status and obesity in adolescents.

\section{Study limitation}

Because of the cross-sectional design and modest sample size of the present study, we could not be certain whether these markers showed ethnic variation in their relation to clinical events such as MS. This would require a much larger prospective study of metabolic disorders in each of several ethnic groups. And due to the difficulties in inviting healthy adolescents to participate in the study, especially scaring of the transrectal ultrasound, uncomfortable and time-consuming OGTT test, there were limit controls fulfill all test.

\section{Conclusions}

Adolescents with PCOS in South China had more metabolic abnormalities than their age- and BMI-matched non-PCOS counterparts. Obesity could worsen insulin resistance, hyperinsulinemia and metabolic syndrome in PCOS adolescents.

\section{Acknowledgements}

This study was supported by National Natural Science Foundation of China (Grant No. 81070466), Science Technology Research Project of Guangdong
Province (Grant No. 2010B031600058), and 2010 Key-projects of Clinical Research of Ministry of Health.

We sincerely appreciate the editing of this manuscript from Dr. Yingzhen Chen at the Department of Anesthesiology, Sun Yat-sen Memorial Hospital.

\section{Author details}

'Department of Obstetrics and Gynecology, Memorial Hospital of Sun YatSen University, Guangzhou, Guangdong, China. ²Department of Obstetrics and Gynecology, The First Affiliated Hospital of Kunming Medical College, Kunming, Yunnan, China.

\section{Authors' contributions}

J.H. performed the study, analyzed and interpreted the data and drafted the paper. R.N. and X.C. performed collected, analyzed the data and revised the draft. L.H. and Y.M. performed guided analysis data and performed the blood sample analysis. D.Y. designed, performed the study and revised the paper. All authors read and approved the final manuscript.

\section{Competing interests}

The authors declare that they have no competing interests.

Received: 19 August 2010 Accepted: 17 November 2010 Published: 17 November 2010

\section{References}

1. Azziz R, Woods KS, Reyna R, Key TJ, Knochenhauer ES, Yildiz BO: The prevalence and features of the polycystic ovary syndrome in an unselected population. J Clin Endocrinol Metab 2004, 89:2745-2749.

2. Revised 2003 consensus on diagnostic criteria and long-term health risks related to polycystic ovary syndrome. Fertil Steril 2004, 81:19-25.

3. Salley KE, Wickham EP, Cheang Kl, Essah PA, Karjane NW, Nestler JE: Glucose intolerance in polycystic ovary syndrome-a position statement of the Androgen Excess Society. J Clin Endocrinol Metab 2007, 92:4546-4556.

4. Moran LJ, Misso ML, Wild RA, Norman RJ: Impaired glucose tolerance, type 2 diabetes and metabolic syndrome in polycystic ovary syndrome: a systematic review and meta-analysis. Hum Reprod Update 2010, 16:347-363.

5. Wild RA, Carmina E, Diamanti-Kandarakis E, Dokras A, Escobar-Morreale HF, Futterweit W, Lobo R, Norman RJ, Talbott E, Dumesic DA: Assessment of cardiovascular risk and prevention of cardiovascular disease in women with the polycystic ovary syndrome: a consensus statement by the Androgen Excess and Polycystic Ovary Syndrome (AE-PCOS) Society. $J$ Clin Endocrinol Metab 2010, 95:2038-2049.

6. Chen X, Yang D, Li L, Feng S, Wang L: Abnormal glucose tolerance in Chinese women with polycystic ovary syndrome. Hum Reprod 2006, 21:2027-2032.

7. Ni RM, Mo Y, Chen X, Zhong J, Liu W, Yang D: Low prevalence of the metabolic syndrome but high occurrence of various metabolic disorders in Chinese women with polycystic ovary syndrome. Eur J Endocrinol 2009, 161:411-418.

8. Diamanti-Kandarakis E: Role of obesity and adiposity in polycystic ovary syndrome. Int J Obes 2007.

9. Oragnisation $\mathrm{WH}$ : Measuring obesity: classification and distribution of anthropometric data. Volume Nutr UD, EUR/ICP/NUT 125. Copenhagen 1989.

10. Chobanian AV, Bakris GL, Black HR, Cushman WC, Green LA, Izzo JL, Jones DW, Materson BJ, Oparil S, Wright JT, Roccella EJ: The Seventh Report of the Joint National Committee on Prevention, Detection, Evaluation, and Treatment of High Blood Pressure: the JNC 7 report. JAMA 2003, 289:2560-2572.

11. Hatch R, Rosenfield RL, Kim MH, Tredway D: Hirsutism: implications, etiology, and management. Am J Obstet Gynecol 1981, 140:815-830.

12. Diagnosis and classification of diabetes mellitus. Diabetes Care 2008, 31(Suppl 1):S55-60.

13. Chen X, Ni R, Mo Y, Li L, Yang D: Appropriate BMI levels for PCOS patients in Southern China. Hum Reprod 2010, 25:1295-1302.

14. Alberti KG, Zimmet $P$, Shaw J: The metabolic syndrome-a new worldwide definition. Lancet 2005, 366:1059-1062.

15. Zimmet P, Alberti G, Kaufman F, Tajima N, Silink M, Arslanian S, Wong G, Bennett P, Shaw J, Caprio S: The metabolic syndrome in children and adolescents. Lancet 2007, 369:2059-2061. 
16. Ehrmann DA: Polycystic ovary syndrome. N Engl J Med 2005, 352:1223-1236.

17. Bridger T, MacDonald S, Baltzer F, Rodd C: Randomized placebo-controlled trial of metformin for adolescents with polycystic ovary syndrome. Arch Pediatr Adolesc Med 2006, 160:241-246.

18. Palmert MR, Gordon CM, Kartashov Al, Legro RS, Emans SJ, Dunaif A: Screening for abnormal glucose tolerance in adolescents with polycystic ovary syndrome. J Clin Endocrinol Metab 2002, 87:1017-1023.

19. Silfen ME, Denburg MR, Manibo AM, Lobo RA, Jaffe R, Ferin M, Levine LS, Oberfield SE: Early endocrine, metabolic, and sonographic characteristics of polycystic ovary syndrome (PCOS): comparison between nonobese and obese adolescents. J Clin Endocrinol Metab 2003, 88:4682-4688.

20. Lee $H$, Oh JY, Sung YA, Chung $H$, Cho WY: The prevalence and risk factors for glucose intolerance in young Korean women with polycystic ovary syndrome. Endocrine 2009, 36:326-332.

21. Nur MM, Newman IM, Siqueira LM: Glucose Metabolism in Overweight Hispanic Adolescents With and Without Polycystic Ovary Syndrome. Pediatrics 2009, 124:E496-E502.

22. Barlow SE: Expert committee recommendations regarding the prevention, assessment, and treatment of child and adolescent overweight and obesity: summary report. Pediatrics 2007, 120(Suppl 4): S164-192.

23. Legro RS, Kunselman AR, Dodson WC, Dunaif A: Prevalence and predictors of risk for type 2 diabetes mellitus and impaired glucose tolerance in polycystic ovary syndrome: a prospective, controlled study in 254 affected women. J Clin Endocrinol Metab 1999, 84:165-169.

24. Ehrmann DA, Barnes RB, Rosenfield RL, Cavaghan MK, Imperial J: Prevalence of impaired glucose tolerance and diabetes in women with polycystic ovary syndrome. Diabetes Care 1999, 22:141-146.

25. Norman RJ, Masters L, Milner CR, Wang JX, Davies MJ: Relative risk of conversion from normoglycaemia to impaired glucose tolerance or noninsulin dependent diabetes mellitus in polycystic ovarian syndrome. Hum Reprod 2001, 16:1995-1998.

26. Legro $R S$, Gnatuk $C L$, Kunselman $A R$, Dunaif $A$ : Changes in glucose tolerance over time in women with polycystic ovary syndrome: a controlled study. J Clin Endocrinol Metab 2005, 90:3236-3242.

27. Coviello AD, Legro RS, Dunaif A: Adolescent girls with polycystic ovary syndrome have an increased risk of the metabolic syndrome associated with increasing androgen levels independent of obesity and insulin resistance. J Clin Endocrinol Metab 2006, 91:492-497.

28. Bhattacharya SM: Metabolic syndrome in females with polycystic ovary syndrome and International Diabetes Federation criteria. J Obstet Gynaecol Res 2008, 34:62-66.

29. Fruzzetti F, Perini $D$, Lazzarini V, Parrini $D$, Genazzani AR: Hyperandrogenemia influences the prevalence of the metabolic syndrome abnormalities in adolescents with the polycystic ovary syndrome. Gynecol Endocrinol 2009, 25:335-343.

30. Goodman E, Daniels SR, Morrison JA, Huang B, Dolan LM: Contrasting prevalence of and demographic disparities in the World Health Organization and National Cholesterol Education Program Adult Treatment Panel III definitions of metabolic syndrome among adolescents. J Pediatr 2004, 145:445-451.

31. Yii MF, Lim CE, Luo X, Wong WS, Cheng NC, Zhan X: Polycystic ovarian syndrome in adolescence. Gynecol Endocrinol 2009, 25:634-639.

32. van Hooff MH, Voorhorst FJ, Kaptein MB, Hirasing RA, Koppenaal C, Schoemaker J: Insulin, androgen, and gonadotropin concentrations, body mass index, and waist to hip ratio in the first years after menarche in girls with regular menstrual cycles, irregular menstrual cycles, or oligomenorrhea. J Clin Endocrinol Metab 2000, 85:1394-1400.

33. Carmina E, Oberfield SE, Lobo RA: The diagnosis of polycystic ovary syndrome in adolescents. Am J Obstet Gynecol 2010, 203(3):201, e1-5. Epub 2010 May 1.

34. Diamanti-Kandarakis E: PCOS in adolescents. Best Pract Res Clin Obstet Gynaecol 2009, 24(2):173-83.

35. Rossi B, Sukalich S, Droz J, Griffin A, Cook S, Blumkin A, Guzick DS, Hoeger KM: Prevalence of metabolic syndrome and related characteristics in obese adolescents with and without polycystic ovary syndrome. J Clin Endocrinol Metab 2008, 93:4780-4786.

36. Broekmans FJ, Knauff EA, Valkenburg O, Laven JS, Eijkemans MJ, Fauser BC: PCOS according to the Rotterdam consensus criteria: Change in prevalence among WHO-II anovulation and association with metabolic factors. BJOG 2006, 113:1210-1217.

37. Diamanti-Kandarakis E, Panidis D: Unravelling the phenotypic map of polycystic ovary syndrome (PCOS): a prospective study of 634 women with PCOS. Clin Endocrinol (Oxf) 2007, 67:735-742.

38. Shroff R, Syrop CH, Davis W, Van Voorhis BJ, Dokras A: Risk of metabolic complications in the new PCOS phenotypes based on the Rotterdam criteria. Fertil Steril 2007, 88:1389-1395.

39. Rosenfield RL: Clinical review: Identifying children at risk for polycystic ovary syndrome. J Clin Endocrinol Metab 2007, 92:787-796.

40. Jeffrey Chang R, Coffler MS: Polycystic ovary syndrome: early detection in the adolescent. Clin Obstet Gynecol 2007, 50:178-187.

41. Salehi M, Bravo-Vera R, Sheikh A, Gouller A, Poretsky L: Pathogenesis of polycystic ovary syndrome: what is the role of obesity? Metabolism 2004, 53:358-376,

42. Toprak S, Yonem A, Cakir B, Guler S, Azal O, Ozata M, Corakci A: Insulin resistance in nonobese patients with polycystic ovary syndrome. Horm Res 2001, 55:65-70.

43. DeUgarte CM, Bartolucci AA, Azziz R: Prevalence of insulin resistance in the polycystic ovary syndrome using the homeostasis model assessment. Fertil Steril 2005, 83:1454-1460.

doi:10.1186/1477-7827-8-142

Cite this article as: Huang et al: Metabolic abnormalities in adolescents with polycystic ovary syndrome in south china. Reproductive Biology and Endocrinology 2010 8:142.

\section{Submit your next manuscript to BioMed Central and take full advantage of:}

- Convenient online submission

- Thorough peer review

- No space constraints or color figure charges

- Immediate publication on acceptance

- Inclusion in PubMed, CAS, Scopus and Google Scholar

- Research which is freely available for redistribution 